\title{
A Fairer Mirror: Equity-limited Healthcare System Rankings
}

\author{
Samuel Reisman 1, 2, Begum Ahmed ${ }^{1}$, Mostafa Balboul ${ }^{1}$, Zev Blumenkranz $^{3}$ \\ ${ }^{1}$ College of Medicine, SUNY Downstate College of Medicine, Brooklyn, New York, United States, ${ }^{2}$ SUNY Downstate School of Public Health, \\ Brooklyn, New York, United States, ${ }^{3}$ New York Medical College, Valhalla, New York, United States
}

Objective

- Describe the diverse determinants of national health and how they are compositely graded in health care system rankings.

- Articulate intrinsic reasons why equity should not be subsumed within other evaluative categories.

- Design an equity-limited ratings framework for limiting maximum ratings of inequitible healthcare systems.

Introduction

Healthcare systems are often evaluated using comparative health care rankings. Simulations have shown that maximally inequitable health care systems can perform well in published, influential health care system rankings by excelling in non-equity categories [1], resulting in highly ranked yet grossly inequitable healthcare systems. Recently, despite below average equity rankings, the healthcare systems of Australia and New Zealand ranked among the top four in The Commonwealth Fund's international comparative study Mirror, Mirror 2017 [2]. Equity rankings should logically limit non-equity rankings given the insignificance of healthcare system improvements to those lacking adequate healthcare coverage. We analyzed whether an equity-limited ranking methodology would limit overall rankings for significantly inequitable healthcare systems while maintaining the general findings of the Commonwealth Fund study.

\section{Methods}

We reanalyzed The Commonwealth Fund's 2017 international health care system comparison using a modified, equity-limited methodology. For each country, maximum non-equity domain summary scores were limited to the equity domain summary score. Countries were ranked using the mean of the five domain-specific performance scores. Overall rankings were compared to the original rankings.

\section{Results}

Seven of eleven countries had an overall rank change in the equity-limited model. Countries with above average overall ratings but poor equity ratings had the greatest changes in overall rank. Australia's overall ranking decreased from second to seventh, thereby matching its equity ranking of seventh. New Zealand changed from fourth to eighth overall, matching its equity ranking as well. Other changes were less significant, with changes of only one overall rank position.

Notably, the bottom three countries and the top country were unchanged.

\section{Conclusions}

Equity-limited ranking methodologies can prevent inequitable health care systems from attaining high overall ratings. Such equitylimited rankings are logical considering the diminished significance of health care system improvements to those lacking adequate health coverage. Methodologies that incorporate equity limits should be used to produce fairer rankings that respect the dignity and rights of all individuals.

\section{References}

1. Reisman S, Blumenkranz Z. Comparative health care system rankings can obscure maximal inequities: A simulation study. Society for Public Health Education (SOPHE) 69th Annual Conference. 2018, June. 
2. Schneider EC, Sarnak DO, Squires D, Shah A, Doty MM. Mirror, Mirror 2017: International Comparison Reflects Flaws and Opportunities for Better U.S. Health Care. The Commonwealth Fund. 2017, July. 